\title{
THE VIBRATION OF AN ELASTIC DIELECTRIC WITH PIEZOELECTROMAGNETISM
}

\author{
BY
}

J. S. YANG (Dept. of Civil Engineering and Operations Research, Princeton University, Princeton, NJ)

AND

X. Y. WU (Dept. of Civil and Environmental Engineering, Carleton University, Ottawa, Ontario, Canada)

\begin{abstract}
This paper presents a few results on the free vibration of a finite elastic dielectric with linear piezoelectromagnetism. Following the proof of selfadjointness, the orthogonality of modes corresponding to different frequencies is proved. A variational principle is given in Rayleigh quotient form for the natural frequency. The variational principle is mixed in the sense that all field variables can be varied independently, and it can be used to generate other variational principles.
\end{abstract}

1. Introduction. The theory of piezoelectromagnetism (dynamic piezoelectricity) has fully dynamic electromagnetic fields. Reciprocity, uniqueness, and minimum principles have been proved in [1]. The vibration of piezoelectromagnetic plates has been studied [2-4] to consider the effect of electromagnetic radiation. The prediction of electromagnetic radiation from vibrating piezoelectric bodies is important in the resonator industry. Knowledge of the amount of energy radiated is needed in computing the quality factor of the resonator. The exact treatment of radiation phenomena requires a fully dynamic theory. The quasi-static theory of piezoelectricity can at most give an approximation of the radiation. A variational principle for piezoelectromagnetism is given in [5], which can be used to derive field equations for piezoelectromagnetism. A mixed variational principle for the field equations of piezoelectromagnetism is given in [6].

In this paper, a few basic properties of the eigenvalue problem for the free vibration of a finite elastic dielectric with linear piezoelectromagnetism are established. The selfadjointness of the eigenvalue problem is proved first, which then leads to the orthogonality of modes corresponding to different frequencies. A mixed variational formulation for the natural frequency is derived in Rayleigh quotient form with all field variables as independent variables. The variational principle can be used to derive other variational principles. This will be shown by an example. The variational principles given here generalize the results in [8] from the quasistatic case to the dynamic case.

Received May 15, 1993.

1991 Mathematics Subject Classification. Primary 73R05.

(C)1995 Brown University 
2. Governing equations. Let the finite spatial region occupied by the piezoelectromagnetic elastic dielectric be $V$, the boundary surface of $V$ be $S$, the unit outward normal of $S$ be $n_{i}$, and $S$ be partitioned in the following ways:

$$
\begin{aligned}
& S_{u} \cup S_{T}=S_{\phi} \cup S_{D}=S_{A} \cup S_{H}=S, \\
& S_{u} \cap S_{T}=S_{\phi} \cap S_{D}=S_{A} \cap S_{H}=\varnothing .
\end{aligned}
$$

The governing equations for the free motion of a finite piezoelectromagnetic body in $V$ are [5]

$$
\begin{gathered}
T_{j i, j}=\rho \dot{v}_{i}, \quad-\rho v_{i}=-\rho \dot{u}_{i} \quad \text { in } V, \\
S_{i j}-\frac{1}{2}\left(u_{j, i}+u_{i, j}\right)=0 \text { in } V, \\
-E_{i}-\phi_{, i}=\dot{A}_{i}, \quad B_{i}-\varepsilon_{i j k} A_{k, j}=0 \text { in } V, \\
D_{i, i}=0, \quad-\varepsilon_{i j k} H_{k, j}=-\dot{D}_{i} \text { in } V, \\
T_{i j}-\left(c_{i j k l} S_{k l}-e_{k i j} E_{k}\right)=0 \text { in } V, \\
-D_{i}-\left(-e_{i k l} S_{k l}-\varepsilon_{i k} E_{k}\right)=0 \text { in } V, \\
H_{i}-\frac{1}{\mu_{0}} B_{i}=0 \text { in } V,
\end{gathered}
$$

with homogeneous boundary conditions

$$
\begin{aligned}
u_{i}=0 & \text { on } S_{u}, & -T_{j i} n_{j}=0 & \text { on } S_{T}, \\
\phi=0 & \text { on } S_{\phi}, & -D_{i} n_{i}=0 & \text { on } S_{D}, \\
\varepsilon_{i j k} n_{j} A_{k}=0 & \text { on } S_{A}, & \varepsilon_{i j k} n_{j} H_{k}=0 & \text { on } S_{H},
\end{aligned}
$$

where $\rho$ is mass density, $T_{i j}$ stress, $S_{i j}$ strain, $u_{i}$ displacement, $v_{i}$ velocity, $E_{i}$ electric field, $D_{i}$ electric displacement, $B_{i}$ magnetic induction, $H_{i}$ magnetic field, $\phi$ and $A_{i}$ the scalar and vector potentials of the electromagnetic fields in the dielectric, and $\mu_{0}$ is the magnetic permeability of free space. $c_{i j k l}, e_{k i j}$, and $\varepsilon_{i j}$ are all material constants. $\varepsilon_{i j k}$ is the permutation tensor. Because of the potential representation $(2)_{3}$ of the electromagnetic fields, only two $\left((2)_{4}\right)$ of the four Maxwell's equations are left and the other two are identically satisfied.

We note that the stress equation of motion $(2)_{1}$ has been written in terms of the velocity $v_{i}$ so that only the first-order time derivative appears. This is for consistency in form with the first-order time derivative in Maxwell's equations and the potential representation of the electromagnetic fields.

The homogeneous electromagnetic boundary conditions $(3)_{2,3}$ include the two common electromagnetic boundary conditions [7] of short circuit boundaries (electric wall, on which tangential $\mathbf{E}$ and normal $\mathbf{B}$ vanish) and open circuit boundary (magnetic wall, on which tangential $\mathbf{H}$ and normal $\mathbf{D}$ vanish) as special cases. 
For time harmonic motions, let

$$
\begin{aligned}
u_{i}(\mathbf{x}, t) & =u_{i}(\mathbf{x}) \cos \omega t, & & v_{i}(\mathbf{x}, t)=v_{i}(\mathbf{x}) \sin \omega t, \\
T_{i j}(\mathbf{x}, t) & =T_{i j}(\mathbf{x}) \cos \omega t, & & S_{i j}(\mathbf{x}, t)=S_{i j}(\mathbf{x}) \cos \omega t, \\
E_{i}(\mathbf{x}, t) & =E_{i}(\mathbf{x}) \cos \omega t, & & D_{i}(\mathbf{x}, t)=D_{i}(\mathbf{x}) \cos \omega t, \\
\phi(\mathbf{x}, t) & =\phi(\mathbf{x}) \cos \omega t, & & \\
H_{i}(\mathbf{x}, t) & =H_{i}(\mathbf{x}) \sin \omega t, & & B_{i}(\mathbf{x}, t)=B_{i}(\mathbf{x}) \sin \omega t, \\
A_{i}(\mathbf{x}, t) & =A_{i}(\mathbf{x}) \sin \omega t . & &
\end{aligned}
$$

Then (2) and (3) become

$$
\begin{aligned}
& T_{j i, j}=\omega \rho v_{i}, \quad-\rho v_{i}=\omega \rho u_{i} \text { in } V, \\
& S_{i j}-\frac{1}{2}\left(u_{j, i}+u_{i, j}\right)=0 \text { in } V, \\
& -E_{i}-\phi_{, i}=\omega A_{i}, \quad B_{i}-\varepsilon_{i j k} A_{k, j}=0 \text { in } V, \\
& D_{i, i}=0, \quad-\varepsilon_{i j k} H_{k, j}=\omega D_{i} \text { in } V, \\
& T_{i j}-\left(c_{i j k l} S_{k l}-e_{k i j} E_{k}\right)=0 \text { in } V, \\
& -D_{i}-\left(-e_{i k l} S_{k l}-\varepsilon_{i k} E_{k}\right)=0 \text { in } V, \\
& H_{i}-\frac{1}{\mu_{0}} B_{i}=0 \text { in } V,
\end{aligned}
$$

and

$$
\begin{array}{rlll}
u_{i}=0 & \text { on } S_{u}, & -T_{j i} n_{j}=0 & \text { on } S_{T}, \\
\phi=0 & \text { on } S_{\phi}, & -D_{i} n_{i}=0 & \text { on } S_{D}, \\
\varepsilon_{i j k} n_{j} A_{k}=0 & \text { on } S_{A}, & \varepsilon_{i j k} n_{j} H_{k}=0 & \text { on } S_{H} .
\end{array}
$$

Values of $\omega$ are sought corresponding to which nontrivial solutions of $u_{i}, v_{i}, S_{i j}$, $T_{i j}, \phi, E_{i}, D_{i}, A_{i}, H_{i}$, and $B_{i}$ exist. Hence (5) and (6) constitute an eigenvalue problem. For (5) and (6), it is convenient to introduce the electric enthalpy density function

$$
\begin{aligned}
H(\mathbf{S}, \mathbf{E}, \mathbf{B})= & \frac{1}{2} c_{i j k l} S_{i j} S_{k l}-e_{i j k} E_{i} S_{j k} \\
& -\frac{1}{2} \varepsilon_{i j} E_{i} E_{j}+\frac{1}{2} \mu_{0}^{-1} B_{i} B_{i} \text { in } V .
\end{aligned}
$$

Then (5) and (6) can be written as

$$
\begin{aligned}
& T_{j i, j}=\omega \rho v_{i}, \quad-\rho v_{i}=\omega \rho u_{i} \quad \text { in } V, \\
& S_{i j}-\frac{1}{2}\left(u_{j, i}+u_{i, j}\right)=0 \quad \text { in } V, \\
& -E_{i}-\phi_{, i}=\omega A_{i}, \quad B_{i}-\varepsilon_{i j k} A_{k, j}=0 \quad \text { in } V, \\
& D_{i, i}=0, \quad-\varepsilon_{i j k} H_{k, j}=\omega D_{i} \text { in } V, \\
& T_{i j}-\frac{\partial H}{\partial S_{i j}}=0, \quad-D_{i}-\frac{\partial H}{\partial E_{i}}=0, \quad H_{i}-\frac{\partial H}{\partial B_{i}}=0 \quad \text { in } V,
\end{aligned}
$$

and

$$
\begin{aligned}
u_{i}=0 & \text { on } S_{u}, & -T_{j i} n_{j}=0 & \text { on } S_{T}, \\
\phi=0 & \text { on } S_{\phi}, & -D_{i} n_{i}=0 & \text { on } S_{D}, \\
\varepsilon_{i j k} n_{j} A_{k}=0 & \text { on } S_{A}, & \varepsilon_{i j k} n_{j} H_{k}=0 & \text { on } S_{H} .
\end{aligned}
$$


3. Selfadjointness and orthogonality. We introduce the following abstract notations of vector $\mathbf{U}$, operators $\mathbf{L}$ and $\mathbf{M}$, and inner product $\langle$,$\rangle :$

$$
\begin{gathered}
\mathbf{U}=\left\{u_{i}, v_{i}, T_{i j}, D_{i}, H_{i}, \phi, A_{i}, S_{i j}, E_{i}, B_{i}\right\}, \\
\mathbf{L} \mathbf{U}=\left\{T_{j i, j},-\rho v_{i}, S_{i j}-\frac{1}{2}\left(u_{j, i}+u_{i, j}\right), D_{i, i},-E_{i}-\phi_{, i},\right. \\
\left.B_{i}-\varepsilon_{i j k} A_{k, j},-\varepsilon_{i j k} H_{k, j}, T_{i j}-\frac{\partial H}{\partial S_{i j}},-D_{i}-\frac{\partial H}{\partial E_{i}}, H_{i}-\frac{\partial H}{\partial B_{i}}\right\}, \\
\mathbf{M U}=\left\{\rho v_{i}, \rho u_{i}, 0, A_{i}, 0,0, D_{i}, 0,0,0\right\}, \\
\left\langle\mathbf{U}, \mathbf{U}^{*}\right\rangle=\int_{V}\left[u_{i} u_{i}^{*}+v_{i} v_{i}^{*}+T_{i j} T_{i j}^{*}+D_{i} D_{i}^{*}+H_{i} H_{i}^{*}\right. \\
\left.+\phi \phi^{*}+A_{i} A_{i}^{*}+S_{i j} S_{i j}^{*}+E_{i} E_{i}^{*}+B_{i} B_{i}^{*}\right] d V,
\end{gathered}
$$

where $\mathbf{U}^{*}$ is another arbitrary abstract vector. It is clear that the above inner product is symmetric, that is, $\left\langle\mathbf{U}, \mathbf{U}^{*}\right\rangle=\left\langle\mathbf{U}^{*}, \mathbf{U}\right\rangle$. With the above definitions, Eq. (8) in $V$ can be written as

$$
\mathbf{L U}=\omega \mathbf{M U} .
$$

It can be verified with integration by parts that for any two abstract vectors $\mathbf{U}$ and $\mathrm{U}^{*}$ satisfying homogeneous boundary conditions (9) the following is true:

$$
\begin{aligned}
& \left\langle\mathbf{L} \mathbf{U}, \mathbf{U}^{*}\right\rangle=\int_{V}\left\{T_{j i, j} u_{i}^{*}-\rho v_{i} v_{i}^{*}+\left[S_{i j}-\frac{1}{2}\left(u_{j, i}+u_{i, j}\right)\right] T_{i j}^{*}\right. \\
& -\left(E_{i}+\phi_{, i}\right) D_{i}^{*}+\left(B_{i}-\varepsilon_{i j k} A_{k, j}\right) H_{i}^{*}+D_{i, i} \phi^{*}-\varepsilon_{i j k} H_{k, j} A_{i}^{*} \\
& \left.+\left(T_{i j}-\frac{\partial H}{\partial S_{i j}}\right) S_{i j}^{*}-\left(D_{i}+\frac{\partial H}{\partial E_{i}}\right) E_{i}^{*}+\left(H_{i}-\frac{\partial H}{\partial B_{i}}\right) B_{i}^{*}\right\} d V \\
& =\int_{V}\left\{u_{i} T_{j i, j}^{*}-v_{i} \rho v_{i}^{*}+T_{i j}\left[S_{i j}^{*}-\frac{1}{2}\left(u_{j, i}^{*}+u_{i, j}^{*}\right)\right]\right. \\
& -D_{i}\left(E_{i}^{*}+\phi_{, i}^{*}\right)+H_{i}\left(B_{i}^{*}-\varepsilon_{i j k} A_{k, j}^{*}\right)+\phi D_{i, i}^{*}-A_{i} \varepsilon_{i j k} H_{k, j}^{*} \\
& \left.+S_{i j}\left(T_{i j}^{*}-\frac{\partial H^{*}}{\partial S_{i j}^{*}}\right)-E_{i}\left(D_{i}^{*}+\frac{\partial H^{*}}{\partial E_{i}^{*}}\right)+B_{i}\left(H_{i}^{*}-\frac{\partial H^{*}}{\partial B_{i}^{*}}\right)\right\} d V \\
& =\left\langle\mathbf{U}, \mathbf{L U}^{*}\right\rangle \text {, } \\
& \left\langle\mathbf{M U}, \mathbf{U}^{*}\right\rangle=\int_{V}\left(\rho v_{i} u_{i}^{*}+\rho u_{i} v_{i}^{*}+A_{i} D_{i}^{*}+D_{i} A_{i}^{*}\right) d V \\
& =\int_{V}\left(u_{i} \rho v_{i}^{*}+v_{i} \rho u_{i}^{*}+D_{i} A_{i}^{*}+A_{i} D_{i}^{*}\right) d V \\
& =\left\langle\mathbf{U}, \mathbf{M U}^{*}\right\rangle \text {. }
\end{aligned}
$$

Hence the operators $\mathbf{L}$ and $\mathbf{M}$ are selfadjoint for abstract vectors satisfying homogeneous boundary conditions (9). With the selfadjointness, we can now proceed to prove the orthogonality of eigenvectors corresponding to different eigenvalues. Let 
$\omega$ and $\omega^{*}$ be two different eigenvalues and let their corresponding eigenvectors be $\mathbf{U}$ and $\mathbf{U}^{*}$; we have

$$
\begin{aligned}
\mathbf{L U} & =\omega \mathbf{M U}, \\
\mathbf{L U}^{*} & =\omega^{*} \mathbf{M} \mathbf{U}^{*} .
\end{aligned}
$$

Taking the inner product of both sides of $(16)_{1}$ by $\mathbf{U}^{*}$ and both sides of $(16)_{2}$ by $\mathbf{U}$, and then subtracting the resulting equations, we obtain

$$
0=\left(\omega-\omega^{*}\right)\left\langle\mathbf{U}^{*}, \mathbf{M U}\right\rangle .
$$

Since $\omega \neq \omega^{*},(17)$ implies the orthogonality condition

$$
\left\langle\mathbf{U}^{*}, \mathbf{M U}\right\rangle=\int_{V}\left(\rho v_{i} u_{i}^{*}+\rho u_{i} v_{i}^{*}+A_{i} D_{i}^{*}+D_{i} A_{i}^{*}\right) d V=0 .
$$

We note that $(18)$ further implies $\left\langle\mathbf{U}^{*}, \mathbf{L U}\right\rangle=0$, which is another form of the orthogonality condition.

3. A variational principle. In this section, we will give a variational formulation for the eigenvalue problem (8) and (9). Different from the variational formulations for the quasi-static case [8] which are for $\omega^{2}$, the following variational principle is for $\omega$. This is consistent with the corresponding variational principle for pure electromagnetic fields of a finite body [9].

Generally, for a fractional functional

$$
\Pi=\frac{\Lambda}{\Gamma}
$$

we have

$$
\delta \Pi=\frac{1}{\Gamma^{2}}(\Gamma \delta \Lambda-\Lambda \delta \Gamma)=\frac{1}{\Gamma}(\delta \Lambda-\Pi \delta \Gamma) .
$$

Therefore, $\delta \Pi=0$ implies

$$
\delta \Lambda-\Pi \delta \Gamma=0 .
$$

Now we consider the following functional of those $\mathbf{U}$ that satisfy (9):

$$
\Pi_{0}(\mathbf{U})=\frac{\frac{1}{2}\langle\mathbf{L U}, \mathbf{U}\rangle}{\frac{1}{2}\langle\mathbf{M U}, \mathbf{U}\rangle} .
$$

With the selfadjointness of $\mathbf{L}$ and $\mathbf{M}$, it can be verified that the stationary condition of $\Pi_{0}$ is

$$
\left\langle\mathbf{L} \mathbf{U}-\Pi_{0} \mathbf{M U}, \delta \mathbf{U}\right\rangle=0 .
$$

Because of the arbitrariness of $\delta \mathbf{U}$, the stationary condition of $\Pi_{0}$ gives (14), with the stationary value of $\Pi_{0}$ as $\omega$. Here the boundary conditions (9) are constraints that must be satisfied by all the admissible vectors $U$ for $\Pi_{0}$. To include boundary conditions (9) as stationary conditions of variations, we can use Lagrange multipliers to release (9). This leads to the following functional $\Pi_{1}$ which has no constraints 
and gives (14) or (8), and (9) as stationary conditions. To be specific, we define

$$
\begin{aligned}
& \Lambda_{1}(\mathbf{u}, \mathbf{v}, \mathbf{T}, \mathbf{D}, \mathbf{H}, \phi, \mathbf{A}, \mathbf{S}, \mathbf{E}, \mathbf{B}) \\
& =\int_{V}\left\{-\frac{1}{2} \rho v_{i} v_{i}+\left[S_{i j}-\frac{1}{2}\left(u_{j, i}+u_{i, j}\right)\right] T_{i j}-\left(E_{i}+\phi_{, i}\right) D_{i}\right. \\
& \left.+\left(B_{i}-\varepsilon_{i j k} A_{k, j}\right) H_{i}-H(\mathbf{S}, \mathbf{E}, \mathbf{B})\right\} d V \\
& +\int_{S_{u}} T_{j i} n_{j} u_{i} d S+\int_{S_{\phi}} D_{i} n_{i} \phi d S+\int_{S_{4}} \varepsilon_{i j k} n_{j} A_{k} H_{i} d S, \\
& \Gamma_{1}(\mathbf{u}, \mathbf{v}, \mathbf{T}, \mathbf{D}, \mathbf{H}, \phi, \mathbf{A}, \mathbf{S}, \mathbf{E}, \mathbf{B})=\int_{V}\left(\rho u_{i} v_{i}+A_{i} D_{i}\right) d V, \\
& \Pi_{1}(\mathbf{u}, \mathbf{v}, \mathbf{T}, \mathbf{D}, \mathbf{H}, \phi, \mathbf{A}, \mathbf{S}, \mathbf{E}, \mathbf{B})=\frac{\Lambda_{1}}{\Gamma_{1}} .
\end{aligned}
$$

Then we have, after integration by parts,

$$
\begin{aligned}
& \delta \Lambda_{1}=\int_{V}\left\{-\rho v_{i} \delta v_{i}+T_{j i, j} \delta u_{i}+D_{i, i} \delta \phi-\varepsilon_{i j k} H_{k, j} \delta A_{i}\right. \\
& +\left[S_{i j}-\frac{1}{2}\left(u_{j, i}+u_{i, j}\right)\right] \delta T_{i j}-\left(E_{i}+\phi_{, i}\right) \delta D_{i}+\left(B_{i}-\varepsilon_{i j k} A_{k, j}\right) \delta H_{i} \\
& \left.+\left(T_{i j}-\frac{\partial H}{\partial S_{i j}}\right) \delta S_{i j}-\left(D_{i}+\frac{\partial H}{\partial E_{i}}\right) \delta E_{i}+\left(H_{i}-\frac{\partial H}{\partial B_{i}}\right) \delta B_{i}\right\} d V \\
& +\int_{S_{u}} u_{i} \delta T_{j i} n_{j} d S-\int_{S_{T}} T_{j i} n_{j} \delta u_{i} d S \\
& +\int_{S_{i,}} \phi \delta D_{i} n_{i} d S-\int_{S_{D}} D_{i} n_{i} \delta \phi d S \\
& +\int_{S_{1}} \varepsilon_{i j k} n_{j} A_{k} \delta H_{i} d S+\int_{S_{I I}} \varepsilon_{i j k} n_{j} H_{k} \delta A_{i} d S, \\
& \delta \Gamma_{1}=\int_{V}\left(\rho u_{i} \delta v_{i}+\rho v_{i} \delta u_{i}+A_{i} \delta D_{i}+D_{i} \delta A_{i}\right) d V .
\end{aligned}
$$

Therefore, $\delta \Pi_{1}=0$ implies

$$
\begin{gathered}
T_{j i, j}=\Pi_{1} \rho v_{i}, \quad-\rho v_{i}=\Pi_{1} p u_{i} \quad \text { in } V, \\
S_{i j}-\frac{1}{2}\left(u_{j, i}+u_{i, j}\right)=0 \quad \text { in } V, \\
-E_{i}-\phi_{, i}=\Pi_{1} A_{i}, \quad B_{i}-\varepsilon_{i j k} A_{k, j}=0 \quad \text { in } V, \\
D_{i, i}=0, \quad-\varepsilon_{i j k} H_{k, j}=\Pi_{1} D_{i} \quad \text { in } V, \\
T_{i j}-\frac{\partial H}{\partial S_{i j}}=0, \quad-D_{i}-\frac{\partial H}{\partial E_{i}}=0, \quad H_{i}-\frac{\partial H}{\partial B_{i}}=0 \quad \text { in } V, \\
u_{i}=0 \quad \text { on } S_{u}, \quad-T_{j i} n_{j}=0 \quad \text { on } S_{T}, \\
\phi=0 \quad \text { on } S_{\phi}, \quad-D_{i} n_{i}=0 \quad \text { on } S_{D}, \\
\varepsilon_{i j k} n_{j} A_{k}=0 \quad \text { on } S_{A}, \quad \varepsilon_{i j k} n_{j} H_{k}=0 \quad \text { on } S_{H} .
\end{gathered}
$$

Comparing (26) with (8) and (9), we conclude that the stationary conditions of $\Pi_{1}$ in (24) give the eigenvalue problem (8) and (9) with the stationary value of $\Pi_{1}$ as $\omega$. 
This variational formulation is of mixed type in the sense that various mechanical and electromagnetic fields can vary independently and there are no constraints.

5. Other variational principles. The variational principles for the vibration of quasi-static piezoelectricity were summarized and systematically developed in [8]. It was shown that for each Legendre transform of the electric enthalpy $H$ there exists a variational principle. The situation is similar for piezoelectromagnetism. Since the electric enthalpy function for piezoelectromagnetism has more variables, there can be more versions of variational principles. We will just show one as an example and not try to exhaust them.

First we introduce a function $M$ from $H$ through Legendre transform as follows:

$$
M(\mathbf{T}, \mathbf{D}, \mathbf{H})=H(\mathbf{S}, \mathbf{E}, \mathbf{B})-T_{i j} S_{i j}+D_{i} E_{i}-H_{i} B_{i},
$$

which generates the constitutive relations in the following form:

$$
S_{i j}=-\frac{\partial M}{\partial T_{i j}}, \quad E_{i}=\frac{\partial M}{\partial D_{i}}, \quad B_{i}=-\frac{\partial M}{\partial H_{i}} .
$$

Then we define

$$
\begin{aligned}
& \Lambda_{2}(\mathbf{u}, \mathbf{v}, \mathbf{T}, \mathbf{D}, \mathbf{H}, \phi, \mathbf{A}) \\
& \quad=\int_{V}\left[-\frac{1}{2} \rho v_{i} v_{i}-\frac{1}{2}\left(u_{j, i}+u_{i, j}\right) T_{i j}-\phi_{, i} D_{i}\right. \\
& \left.\quad-\varepsilon_{i j k} A_{k, j} H_{i}-M(\mathbf{T}, \mathbf{D}, \mathbf{H})\right] d V \\
& \quad+\int_{S_{u}} T_{j i} n_{j} u_{i} d S+\int_{S_{\phi}} D_{i} n_{i} \phi d S+\int_{S_{i}} \varepsilon_{i j k} n_{j} A_{k} H_{i} d S, \\
& \Gamma_{2}(\mathbf{u}, \mathbf{v}, \mathbf{T}, \mathbf{D}, \mathbf{H}, \phi, \mathbf{A})=\int_{V}\left(\rho u_{i} v_{i}+A_{i} D_{i}\right) d V, \\
& \Pi_{2}(\mathbf{u}, \mathbf{v}, \mathbf{T}, \mathbf{D}, \mathbf{H}, \phi, \mathbf{A})=\frac{\Lambda_{2}}{\Gamma_{2}} .
\end{aligned}
$$

Then we have, after integration by parts,

$$
\begin{aligned}
\delta \Lambda_{2}= & \int_{V}\left\{-\rho v_{i} \delta v_{i}+T_{j i, j} \delta u_{i}+D_{i, i} \delta \phi-\varepsilon_{i j k} H_{k, j} \delta A_{i}\right. \\
& \left.-\left[\frac{\partial M}{\partial T_{i j}}+\frac{1}{2}\left(u_{j, i}+u_{i, j}\right)\right] \delta T_{i j}-\left(\frac{\partial M}{\partial E_{i}}+\phi_{, i}\right) \delta D_{i}-\left(\frac{\partial M}{\partial H_{i}}+\varepsilon_{i j k} A_{k, j}\right) \delta H_{i}\right\} \\
& +\int_{S_{u}} u_{i} \delta T_{j i} n_{j} d S-\int_{S_{T}} T_{j i} n_{j} \delta u_{i} d S \\
& +\int_{S_{\phi}} \phi \delta D_{i} n_{i} d S-\int_{S_{D}} D_{i} n_{i} \delta \phi d S \\
& +\int_{S_{A}} \varepsilon_{i j k} n_{j} A_{k} \delta H_{i} d S+\int_{S_{H}} \varepsilon_{i j k} n_{j} H_{k} \delta A_{i} d S, \\
\delta \Gamma_{2}= & \int_{V}\left(\rho u_{i} \delta v_{i}+\rho v_{i} \delta u_{i}+A_{i} \delta D_{i}+D_{i} \delta A_{i}\right) d V .
\end{aligned}
$$


Therefore, $\delta \Pi_{2}=0$ implies

$$
\begin{aligned}
& T_{j i, j}=\Pi_{2} \rho v_{i}, \quad-\rho v_{i}=\Pi_{2} \rho u_{i} \text { in } V, \\
& -\frac{\partial M}{\partial T_{i j}}-\frac{1}{2}\left(u_{j, i}+u_{i, j}\right)=0 \quad \text { in } V, \\
& -\frac{\partial M}{\partial D_{i}}-\phi_{, i}=\Pi_{2} A_{i}, \quad-\frac{\partial M}{\partial H_{i}}-\varepsilon_{i j k} A_{k, j}=0 \text { in } V, \\
& D_{i, i}=0, \quad-\varepsilon_{i j k} H_{k, j}=\Pi_{2} D_{i} \quad \text { in } V, \\
& u_{i}=0 \quad \text { on } S_{u}, \quad-T_{j i} n_{j}=0 \quad \text { on } S_{T}, \\
& \phi=0 \quad \text { on } S_{\phi}, \quad-D_{i} n_{i}=0 \text { on } S_{D}, \\
& \varepsilon_{i j k} n_{j} A_{k}=0 \quad \text { on } S_{A}, \quad \varepsilon_{i j k} n_{j} H_{k}=0 \text { on } S_{H} .
\end{aligned}
$$

Hence, the stationary conditions of $\Pi_{2}$ give the eigenvalue problem (31), with the stationary value of $\Pi_{2}$ as the eigenvalue. It can be seen that the elimination of $\mathbf{S}$, $\mathbf{E}$, and $\mathbf{B}$ in (8) through the constitutive relation $(8)_{5}$ results in (31). Hence (31) is equivalent to the original eigenvalue problem (8) and (9). The variational principles for $\Pi_{1}$ and $\Pi_{2}$ here generalize indirectly those in [8] to the dynamic case.

\section{REFERENCES}

[1] D. Iesan, Reciprocity, uniqueness and minimum principles in the linear theory of piezoelectricity, Internat. J. Engrg. Sci. (28) 11, 1139-1149 (1990)

[2] R. D. Mindlin, Electromagnetic radiation from a vibrating quartz plate, Internat. J. Solids Structures 9, 697-702 (1973)

[3] P. C. Y. Lee, Electromagnetic radiation from an AT-cut quartz plate under lateral field excitation, J. Appl. Phys. (65) 4, 1395-1399 (1989)

[4] P. C. Y. Lee, Y.-G. Kim, and J. H. Prevost, Electromagnetic radiation from doubly rotated piezoelectric crystal plates at thickness frequencies, J. Appl. Phys. (67) 11, 6633-6642 (1990)

[5] P. C. Y. Lee, $A$ variational principle for the equations of piezoelectromagnetism in elastic dielectric crystals, J. Appl. Phys. (69) 11, 7470-7473 (1991)

[6] J. S. Yang, A generalized variational principle for piezoelectromagnetism in an elastic medium, Arch. Mech. (43) 6, 795-798 (1991)

[7] H.-Y. Yee, An Investigation of Microwave Dielectric Resonators, Internal Memorandum, Microwave Laboratory, W. W. Hansen Laboratories of Physics, Stanford University, Stanford, California, 1963

[8] J. S. Yang, Variational formulations for the vibration of a piezoelectric body, Quart. Appl. Math. 53, 95-104 (1995)

[9] A. D. Berk, Variational principles for electromagnetic resonators and waveguides, IRE Trans. on Antennas and Propagation, AP-4, 1956, pp. 104-111 